\title{
ULTRASONIC INTERACTION STUDIES OF TERNARY MIXTURE SOLUTIONS AT 303 AND 313 TEMPERATURE
}

\author{
Yasmin Akhtar ${ }^{* 1}$ 四 \\ ${ }^{* 1}$ Department of Chemistry, University of Tabuk, Alwajh College, Saudi Arabia
}

DOI: https://doi.org/10.29121/ijetmr.v7.i9.2020.750

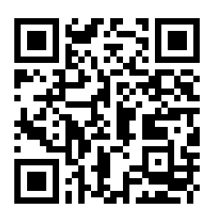

Article Citation: Yasmin Akhtar. (2020). ULTRASONIC INTERACTION STUDIES OF TERNARY MIXTURE SOLUTIONS AT 303 AND 313 TEMPERATURE. International Journal of Engineering Technologies and Management Research, 7(9), 10-13.

https://doi.org/10.29121/ijetmr.v7 i9.2020.750

Published Date: 15 September 2020

Keywords:

Aniline

Cyclohexane

Dimethyl Ketone

Ethyl Methyl Ketone

Adiabatic Compressibility Free

Length

Acoustic Impedance

\begin{abstract}
Densities and ultrasonic velocities of Aniline in cyclohexane + Dimethyl ketone and Aniline in cyclohexane + ethyl methyl ketone of these ternary mixtures of different compositions have been determined experimentally at 303 and $313 \mathrm{~K}$. The results obtained from density and ultrasonic velocity measurement have been used to calculate the acoustical parameters such as, adiabatic compressibility (Ks), free Length (Lf) , and acoustic impedance (Z). The results are discussed in terms of the intermolecular hydrogen bonding and molecular association in these ternary systems.
\end{abstract}

\section{INTRODUCTION}

We present in this paper, the study of interaction between Aniline in cyclohexane + Dimethyl ketone and Aniline in cyclohexane + ethyl methyl ketone of these ternary mixtures. Volumetric and acoustic properties of procainamide hydrochloric acid in aqueous amino acids and ternary mixtures at different temperature. To understand the energy basis of molecular interactions utilizing various thermodynamic methods, volumetric and acoustic studies are vital early development process of any drugs toward an optimal energy interaction profile while retaining a good pharmacological assay [1] Ultrasonic investigation in aqueous solution of electrolytes and non-electrolytes with amino acids provides useful information of liquid systems, because intermolecular and intramolecular association, complex formation and related structural changes affect the compressibility of the system. Ultrasonic wave have been used by many researchers to investigate the nature of molecular interactions and physico-chemical behavior of pure, binary and ternary liquid mixtures. There has been an increased interest in the physicochemical properties of amino acids in aqueous as well as aqueous and non-aqueous electrolytes media to understand the role played by the biological molecules in living organism [2], [3], [4], [5]. Amino acids have zwitter- ion and are the constituents of the most important class of biopolymers, i.e. Proteins. Disarrangement water and electrolyte balance in living

(C) 2020 The Author(s). This is an open access article distributed under the terms of the Creative Commons Attribution License, which permits unrestricted use, distribution, and reproduction in any medium, provided the original author and source are credited. 
Ultrasonic Interaction Studies of Ternary Mixture Solutions at 303 and 313 Temperature

systems cause a wide variety of health problems. Aniline molecule is highly polar and self-associated through hydrogen bonding of their amine group. Being aromatic aniline with amino group is comparatively a strong donor. The $\mathrm{H}$ atoms in the $\mathrm{NH}_{2}$ group can also play the role of electron acceptors.[6]. It is used in the manufacturing of synthetic dyes, drugs and as an accelerator in vulcanization of rubber [7]. The L- valine is a branched chain amino acid which is important for supplying energy to muscles. The branched chain amino acids enhance energy, increase endurance and aid in muscle tissue recovery and repair [8]. The dimethyl ketone, and ethyl methyl ketone are aprotic in nature and belong to same homologous series, especially they have aliphatic nature and have common carbonyl group $(\mathrm{C}=0)$ functional group [8]. Recently we have made systematic effort to investigate the volumetric properties of amino acids in concentrated electrolytic solution [9]. The volumetric studies have been used to the solute -solute and solute-solvent interaction in these ternary systems. This suggests D (+) Lactose in aqueous sodium butyrate solution is strong structure maker than $\mathrm{D}(+)$ galactose in aqueous sodium butyrate solution. These parameters reflect that the solute-co solute interactions are dominating over solute-solvent interactions for the saccharides in aqueous sodium butyrate solutions. [10]

In the present paper, we report densities, $(\rho)$ and ultrasonic velocity (u) of Aniline in cyclohexane + Dimethyl ketone and Aniline in cyclohexane + ethyl methyl ketone of these ternary mixtures of different compositions have been determined experimentally at 303 and $313 \mathrm{~K}$. From these experimental data a number of acoustical parameters namely, adiabatic compressibility $\left(\mathrm{K}_{\mathrm{s}}\right.$ ), free Length $\left(\mathrm{L}_{\mathrm{f}}\right.$ ) and acoustic impedance $(\mathrm{Z})$ respectively have been calculated. These parameters were utilized to study various interactions taking place in ternary mixtures at 303 and $313 \mathrm{~K}$.

\section{EXPERIMENTAL}

Aniline (99.8\% purity), cyclohexane, (99.9\% purity) Dimethyl ketone (99.9\% purity) and ethyl methyl ketone (99.9\% purity) were procured from E. Merck (Germany) and S d Fine Ltd. All the ternary mixtures were prepared in terms of mole fractions from (0.00-0.70). The densities, $\rho$ and ultrasonic velocity $u$ of Aniline in cyclohexane + Dimethyl ketone and Aniline in cyclohexane + ethyl methyl ketone of these ternary mixtures of different compositions have been determined experimentally.

The densities were measured with a single capillary pycnometer (made of Borosil glass) of bulb capacity of $8 \times 10^{-6} \mathrm{~m}^{3}$. The marks of the stems were calibrated using triple distilled water at 303and $313 \mathrm{~K}$. The pycnometer was

kept for about 30 minutes in a thermostatic water bath so that the thermal fluctuation in density was minimized. The ultrasonic velocities in solutions were measured using a single crystal variable path interferometer at $2 \mathrm{MHz}$.

The ultrasonic velocities in ternary mixtures were measured using a single crystal variable path interferometer at $2 \mathrm{MHz}$. The temperature of the test solutions was maintained at 303 and $313 \pm 0.02 \% \mathrm{~K}$ in an electronically controlled thermostatic water bath. The density and velocity data were found to be accurate within $\pm 0.01 \%$ and \pm $0.10 \%$ respectively.

\section{RESULTS AND DISCUSSION}

The densities $(\rho)$ and ultrasonic velocities $(u)$ of Aniline in cyclohexane + Dimethyl ketone and Aniline in cyclohexane + ethyl methyl ketone of these ternary mixtures of different compositions have been determined experimentally at 308 and $313 \mathrm{~K}$ are recorded in Table 1. It is observed that densities $(\rho)$ and ultrasonic velocities $(\mathrm{u})$ for all the ternary systems increase with increase in mole fraction of Aniline. The values of $\rho$ and $u$ increase with increase in concentration of Aniline in all the ternary systems under investigation, which appear to be due to hydrophobic properties of solutes i.e. H-bond forming. The density and ultrasonic velocity decrease with increase in temperature which indicates the changes in structure occurring in the mixtures as a result of $\mathrm{H}$-bond formation lead to decrease in intermolecular forces [11] due to increase in thermal vibration of the ternary system, which causes an increase in volume expansion and hence increase in free path length. Solute may occupy the interstitial spaces in solvent or get solvated forming new weaker bonds. It was suggested [12], [13], [14] that what is experimentally observed for any system, reflects the compromise between the tendency for the ion and the peptide to interact with each other and inclination of the solutes to associate with the solvent. In other cases the values of aliphatic ketones without aniline is much lower, which means, when the mole fraction of aniline increases with increasing trend is 
observed in density and ultrasonic velocity. It result that the addition of aniline increase the effective molecular space due to the substitution of a cyclic aniline by replacing cyclohexane.

The behavior of Aniline considered the following interactions:

- The weak dipolar interaction between ketones and cyclohexane.

- The Aniline is high dielectric constant and basically electron donor nature, when it mixed with ketones there is significant type of dipole-dipole interactions and $\mathrm{H}$ - bonding formation.

- The overlap of hydration groups co spheres of terminal (- $\mathrm{NH}_{3}+$ and $\left.\mathrm{COO}^{-}\right)$and the adjacent groups result in volume change.

The adiabatic compressibility $\left(\mathrm{K}_{\mathrm{s}}\right)$ and Intermolecular free length $\left(\mathrm{L}_{\mathrm{f}}\right)$ of the Aniline in cyclohexane + Dimethyl ketone and Aniline in cyclohexane + ethyl methyl ketone of these ternary mixtures of different compositions have been determined from the density and velocity data at 308 and $313 \mathrm{~K}$. The adiabatic compressibility and Intermolecular free length $\left(\mathrm{L}_{\mathrm{f}}\right)$ was calculated by the equation $(1)$ and $(2)$.

$$
\begin{aligned}
& \mathrm{K}_{\mathrm{s}}=1 / \mathrm{u}^{2} \rho \\
& \mathrm{L}_{\mathrm{f}}=\mathrm{K}_{\mathrm{T}}\left(\mathrm{K}_{\mathrm{s}}\right)^{1 / 2}
\end{aligned}
$$

The per usual of Table 2 exhibits the values of the adiabatic compressibility, Ks and Intermolecular free length $\left(\mathrm{L}_{\mathrm{f}}\right)$ to be decrease with increasing concentration of the systems. Such a decrease in adiabatic compressibility and Intermolecular free length $\left(\mathrm{L}_{\mathrm{f}}\right)$ observed in the ternary systems clearly confirms the conclusion drawn from the ultrasonic velocity data. It is well known fact that when a solute dissolve in a solvent there is decrease interaction of solute and solvent molecules with decrease concentration of ketones. So, the solutions become harder to compress. This will lead to the decrease in compressibility values. This may further ascribed that such a decrease in adiabatic compressibility, $\mathrm{K}_{\mathrm{s}}$ is attributed to the influence of the electrostatic field of the ions $\left(\mathrm{NH}_{3}{ }^{+}\right)$on the accompanying molecules. It may be inferred that the weakening of hydrogen bond strength formed by the solute and solvent molecules and complex formation may also be the reason for decrease in compressibility. The cluster between aniline and ketones molecules is broken up by the interacting molecule, cyclohexane. The intermolecular force of attraction are less between solute and solvent due to this decrease the free length in this ternary systems. Thus is in accordance with the view proposed by others ( M. Iqbal et al 1990).

The values of acoustic impedance, $\mathrm{Z}$ of these ternary systems were calculated by the following relation.

$$
\mathrm{Z}=\mathrm{u} \cdot \rho
$$

The values of $\mathrm{Z}$ increase with increase in different compositions of the Aniline in cyclohexane + Dimethyl ketone and Aniline in cyclohexane + ethyl methyl ketone of these ternary mixtures which indicates solute-solvent interaction are present and they behave as structure makers are shown in Table 2. The specific acoustic impedance of the medium for the particular type of wave being propagated and through it. The transmission of acoustic wave from one medium to another is important for transmission phenomenon in fluids. It may also correlated with ionic size of ions. A definite correlation the specific acoustic impedance and dielectric constant of the solvent medium. The behavior of acoustic impedance can be explained on the basis of lyophobic interaction between solute and solvent molecules, complex ion formation and become responsible for the propagation of ultrasonic wave.

\section{CONCLUSION}

In the present work the weak dipolar interaction between ketones and cyclohexane. The Aniline is high dielectric constant and basically electron donor nature, when it mixed with ketones there is significant type of dipole-dipole interactions and $\mathrm{H}$ - bonding formation. Aniline in cyclohexane + Dimethyl ketone and Aniline in cyclohexane + ethyl methyl ketone of these ternary mixtures which indicates solute-solvent interaction are present and they behave as structure makers. The presence of the molecular association and intermolecular hydrogen bonding of these ternary mixtures. 


\section{SOURCES OF FUNDING}

None.

\section{CONFLICT OF INTEREST}

None.

\section{ACKNOWLEDGMENT}

I am highly thankful to provide the laboratory facilities for research Department of Chemistry, University of Tabuk, K.S.A.

\section{REFERENCES}

[1] D. M. Bhattacharya, D.V. Kawadkar, C.V. Pandhumaker and A. V. Wankahade, J. Chem. Eng. Data, 62(12) (2017) 4083-4092.

[2] Y. Akhtar, International J. Res. Granth. 6(9) (2018) 356-363., Y. Akhtar, International J. Engg. Sci. Res. Tech. 7 (10) (2018) 51-60.

[3] F. J. Millero, A. L. Surdo and C. Shiv, J. Phys. Chem. 87 (1978) 784.

[4] Y. Akhtar, S. F. Ibrahim, Arabian J. Chem. 4 (2011) 487-490; Vishnu D., Y. Akhtar, J. D.Pandey, Acta Acoust. 84(1998) 976.

[5] Y. Akhtar, Fluid Phase Equil. 258 (2007)125. Yasmin Akhtar, October (2017) International J. Res. Granth. 5(10) $160-$

[6] X. Jiang, C. Zhu and Y. Ma, J. Chem. Thermodyn. 71(2014)50-63.

[7] M. S. Hussain, T.K Kabriaz, M. N. Islam and M. E. Haque, J. Chem. Thermodyn. 71 (2014)6-13.

[8] Riyazuddin and M. A. Usmani, Thermochemica. Acta, 527 (2012),112-117.

[9] C. Zhu and X. Jiang, Y. Ma, J. Chem. Thermodyn. 71(2014)50-63.

[10] Y. Akhtar, International J. Engg. Tech. Mang. Res 6 (11) (2019) 11-17.

[11] T. S. Banipal and G. Singh Indian J. Chem. 3A (2000)1011.

[12] A. P. Mishra and Gautum S. K., Indian J. Chem. 40A (2001), 100 and D. L. Q. Yu, Y. Y. Wang and D. Sun Indian J. Chem. 41A (2002)1126.

[13] R. J. Laurich, C. R. Torok and M. J. Tubergen J. Phys. Chem.A 106 (2002)8013.

[14] M. Iqbal, M. Mateullah Can. J. Chem. 68 (1990)7. 